\title{
Harmonious Coloring on Subclasses of Colinear Graphs
}

\author{
Kyriaki Ioannidou and Stavros D. Nikolopoulos \\ Department of Computer Science, University of Ioannina \\ GR-45110 Ioannina, Greece \\ \{kioannid, stavros\}@cs.uoi.gr
}

\begin{abstract}
Given a simple graph $G$, a harmonious coloring of $G$ is a proper vertex coloring such that each pair of colors appears together on at most one edge. The harmonious chromatic number is the least integer $k$ for which $G$ admits a harmonious coloring with $k$ colors. Extending previous NP-completeness results of the harmonious coloring problem on subclasses of chordal and co-chordal graphs, we prove that the problem remains NP-complete for split undirected path graphs; we also prove that the problem is NP-complete for colinear graphs by showing that split undirected path graphs form a subclass of colinear graphs. Moreover, we provide a polynomial solution for the harmonious coloring problem for the class of split strongly chordal graphs, the interest of which lies on the fact that the problem has been proved to be NP-complete on both split and strongly chordal graphs.
\end{abstract}

Keywords: Harmonious coloring, colinear coloring, colinear graphs, split graphs, undirected path graphs, strongly chordal graphs, complexity.

\section{Introduction}

A harmonious coloring of a simple graph $G$ is a proper vertex coloring such that each pair of colors appears together on at most one edge, while the harmonious chromatic number $h(G)$ is the least integer $k$ for which $G$ admits a harmonious coloring with $k$ colors [5].

Several NP-complete problems on arbitrary graphs admit polynomial solutions when restricted to the classes of strongly chordal graphs and undirected path graphs and, thus, interval graphs (see e.g. $[12,18]$ ). However, the harmonious coloring problem, which is NP-hard on arbitrary graphs [22], remains NPcomplete even when restricted to graphs that are simultaneously interval and cographs [3]. More specifically, Bodlaender [3] provides a proof that establishes the NP-completeness of the harmonious coloring problem for disconnected interval graphs and cographs. Recently, we extended Bodlaender's results by showing that the problem remains NP-complete for connected interval graphs [1]. Note that the problem of determining the harmonious chromatic number of connected

\footnotetext{
* This research is co-financed by E.U.-European Social Fund (80\%) and the Greek Ministry of Development-GSRT (20\%).
} 
cographs is trivial, since in such a graph each vertex must receive a distinct color as it is at distance at most 2 from all other vertices [5]. Therefore, the harmonious coloring problem has been proved to be NP-complete on the class of interval graphs and, thus, on the classes of strongly chordal and undirected path graphs.

Additionally, the NP-completeness of the problem has been also proved for the classes of split graphs [1], trees and disconnected bipartite permutation graphs $[9,10]$, connected bipartite permutation graphs [2], and disconnected quasi-threshold graphs [2]. Since the problem of determining the harmonious chromatic number of a connected cograph is trivial, the harmonious coloring problem is polynomially solvable on connected quasi-threshold graphs and threshold graphs.

In this paper we study the complexity status of the harmonious coloring problem on two subclasses of colinear graphs $[17,16]$. We first show that the harmonious coloring problem is NP-complete on split undirected path graphs and, then, we show that the class of split undirected path graphs forms a subclass of colinear graphs; thus, we obtain the NP-completeness of the harmonious coloring problem on colinear graphs as well. Moreover, we provide a polynomial solution for the harmonious coloring problem on split strongly chordal graphs, the interest of which lies on the fact that the problem is NP-complete on both split graphs and strongly chordal graphs $[1,3]$. However, the complexity status of the problem for the class of connected linear graphs still remains an open question; note that the harmonious coloring problem is NP-complete on disconnected linear graphs, since it is NP-complete on disconnected quasi-threshold graphs [2] and quasi-threshold graphs form a subclass of linear graphs $[17,16]$.

\section{Background Results}

In this section we provide some basic graph theory definitions and give some background results on colinear coloring, colinear graphs, and linear graphs. For basic definitions in graph theory refer to $[4,15]$, and for more details on colinear coloring, colinear and linear graphs refer to $[17,16]$.

\subsection{Preliminaries}

Let $G$ be a finite undirected graph with no loops or multiple edges. We denote by $V(G)$ and $E(G)$ the vertex set and edge set of $G$. An edge is a pair of distinct vertices $x, y \in V(G)$, and is denoted by $x y$ if $G$ is an undirected graph and by $\overrightarrow{x y}$ if $G$ is a directed graph. For a set $A \subseteq V(G)$ of vertices of the graph $G$, the subgraph of $G$ induced by $A$ is denoted by $G_{A}$ or $G[A]$. Additionally, the cardinality of a set $A$ is denoted by $|A|$. The set $N(v)=\{u \in V(G): u v \in E(G)\}$ is called the open neighborhood of the vertex $v \in V(G)$ in $G$, sometimes denoted by $N_{G}(v)$ for clarity reasons. The set $N[v]=N(v) \cup\{v\}$ is called the closed neighborhood of the vertex $v \in V(G)$ in $G$. Also, by $\bar{G}$ we denote the complement graph of a graph $G$. 
The greatest integer $r$ for which a graph $G$ contains an independent set of size $r$ is called the independence number or otherwise the stability number of $G$ and is denoted by $\alpha(G)$. The cardinality of the vertex set of the maximum clique in $G$ is called the clique number of $G$ and is denoted by $\omega(G)$. A proper vertex coloring of a graph $G$ is a coloring of its vertices such that no two adjacent vertices are assigned the same color. The chromatic number $\chi(G)$ of $G$ is the least integer $k$ for which $G$ admits a proper vertex coloring with $k$ colors. For the numbers $\omega(G)$ and $\chi(G)$ of an arbitrary graph $G$ the inequality $\omega(G) \leq \chi(G)$ holds. In particular, $G$ is a perfect graph if the equality $\omega\left(G_{A}\right)=\chi\left(G_{A}\right)$ holds $\forall A \subseteq V(G)$.

Next, definitions of some graph classes mentioned throughout the paper follow. A graph is called a chordal graph if it does not contain an induced subgraph isomorphic to a chordless cycle of four or more vertices. A graph is called a co-chordal graph if it is the complement of a chordal graph [15]. A hole is a chordless cycle $C_{n}$ if $n \geq 5$; the complement of a hole is an antihole. Threshold graphs are defined as those graphs where stable subsets of their vertex sets can be distinguished by using a single linear inequality. Threshold graphs were introduced by Chvátal and Hammer [7] and characterized as $\left(2 K_{2}, P_{4}, C_{4}\right)$-free. Quasi-threshold graphs are characterized as the $\left(P_{4}, C_{4}\right)$-free graphs and are also known in the literature as trivially perfect graphs [15].

A graph $G$ is a split graph if there is a partition of the vertex set $V(G)=K+I$, where $K$ induces a clique in $G$ and $I$ induces an independent set. Split graphs are characterized as $\left(2 K_{2}, C_{4}, C_{5}\right)$-free [15]. A chordal graph is an undirected path graph if it is the vertex intersection graph of undirected paths in a tree [14, 20,21]. A graph is strongly chordal if it admits a strong elimination ordering. Strongly chordal graphs were introduced by Farber in [11] and are characterized completely as those chordal graphs which contain no $k$-sun as an induced subgraph (for the definition of a $k$-sun see Section 4 ).

\subsection{Colinear Coloring and Colinear Graphs}

Motivated by the definition of linear coloring on simplicial complexes associated to graphs, first introduced in the context of algebraic topology [8], we recently introduced the colinear coloring on graphs [17].

Definition 1. Let $G$ be a graph and let $v \in V(G)$. The clique set of a vertex $v$ is the set of all maximal cliques of $G$ containing $v$ and is denoted by $\mathcal{C}_{G}(v)$.

Definition 2. Let $G$ be a graph and let $k$ be an integer. A surjective map $\kappa$ : $V(G) \rightarrow\{1,2, \ldots, k\}$ is called a $k$-colinear coloring of $G$ if the collection $\left\{\mathcal{C}_{G}(v)\right.$ : $\kappa(v)=i\}$ is linearly ordered by inclusion for all $i \in\{1,2, \ldots, k\}$. Equivalently, for two vertices $v, u \in V(G)$, if $\kappa(v)=\kappa(u)$ then either $\mathcal{C}_{G}(v) \subseteq \mathcal{C}_{G}(u)$ or $\mathcal{C}_{G}(v) \supseteq \mathcal{C}_{G}(u)$. The least integer $k$ for which $G$ is $k$-colinear colorable is called the colinear chromatic number of $G$ and is denoted by $\lambda(G)$.

The interest to provide boundaries for the chromatic number $\chi(G)$ of an arbitrary graph $G$ through the study of different simplicial complexes associated 


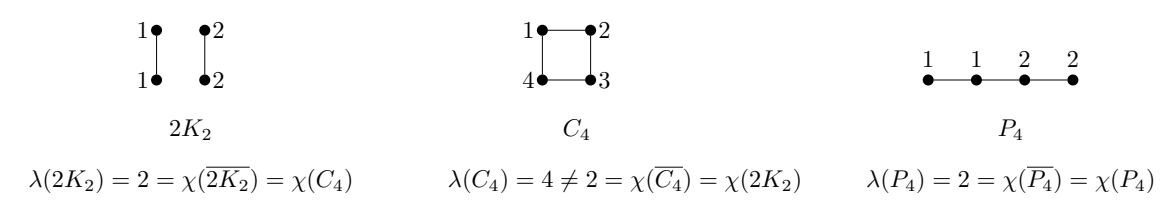

Fig. 1. Illustrating a colinear coloring of the graphs $2 K_{2}, C_{4}$ and $P_{4}$ with the least possible colors.

to $G$, which is found in algebraic topology bibliography, drove the motivation for studying the relation between the chromatic number $\chi(G)$ and the colinear chromatic number $\lambda(\bar{G})$. In Figure 1 we depict a colinear coloring of the well known graphs $2 K_{2}, C_{4}$ and $P_{4}$, using the least possible colors, and show the relation between the chromatic number $\chi(G)$ of each graph $G \in\left\{2 K_{2}, C_{4}, P_{4}\right\}$ and the colinear chromatic number $\lambda(\bar{G})$.

In [17] we presented a polynomial time algorithm for colinear coloring which can be applied to any graph $G$ and, also, we proved the following results.

Proposition 1. ([17]) For any graph $G, \lambda(\bar{G}) \geq \chi(G)$.

Proposition 2. ([17]) Let $G$ be a graph. A coloring $\kappa: V(G) \rightarrow\{1,2, \ldots, k\}$ of $G$ is a k-colinear coloring of $G$ if and only if either $N_{G}[u] \subseteq N_{G}[v]$ or $N_{G}[u] \supseteq$ $N_{G}[v]$ holds in $G$, for every $u, v \in V(G)$ with $\kappa(u)=\kappa(v)$.

Motivated by these results and the Perfect Graph Theorem [15], we studied those graphs for which the equality $\chi(G)=\lambda(\bar{G})$ holds for every induced subgraph and characterized known graph classes in terms of the $\chi$-colinear and the $\alpha$-colinear properties [17]. Moreover, it was interesting to study those graphs which are characterized completely by the $\chi$-colinear or the $\alpha$-colinear property. The outcome of this study was to conclude that these graphs form two new classes of perfect graphs, which we call colinear and linear graphs, respectively [16].

Definition 3. A graph $G$ is called colinear if and only if $\chi\left(G_{A}\right)=\lambda\left(\bar{G}_{A}\right), \forall A \subseteq$ $V(G)$. A graph $G$ is called linear if and only if $\alpha\left(G_{A}\right)=\lambda\left(G_{A}\right), \forall A \subseteq V(G)$.

We also showed inclusion relations between the classes of colinear and linear graphs and other subclasses of co-chordal and chordal graphs [16]. More specifically, the class of colinear graphs is a subclass of co-chordal graphs, a superclass of threshold graphs, and is distinguished from the class of split graphs. Additionally, linear graphs form a subclass of chordal graphs and a superclass of quasi-threshold graphs. We also proved that any $P_{6}$-free strongly chordal graph is a linear graph.

The inclusion relations among the classes of colinear graphs, linear graphs, and other subclasses of co-chordal and chordal graphs are depicted in Figure 2. 


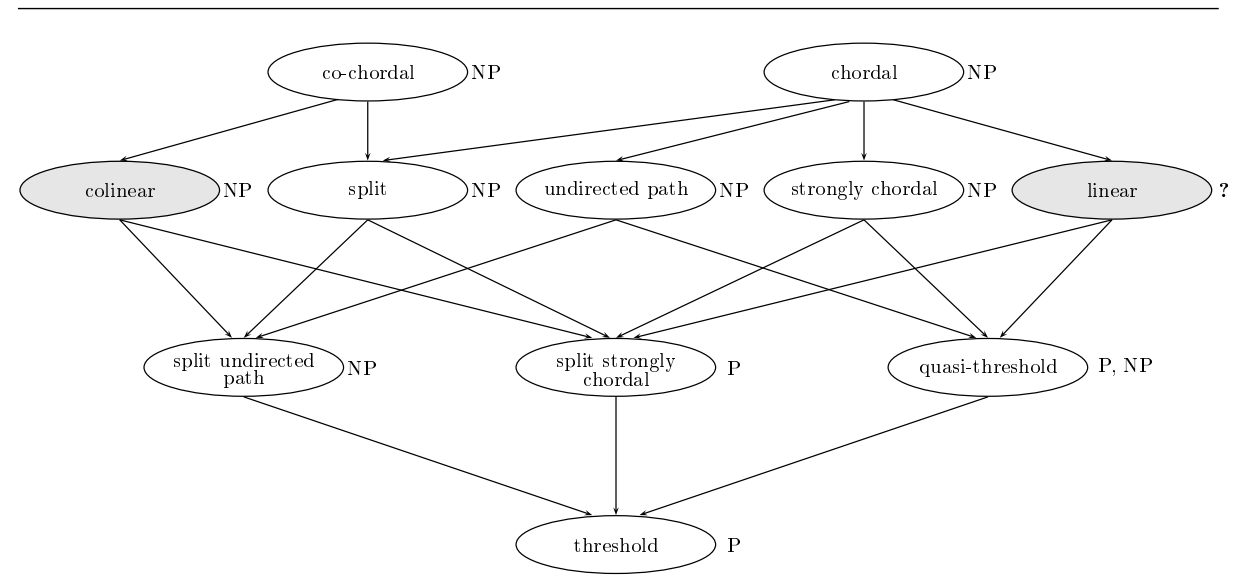

Fig. 2. Illustrating the complexity status of the harmonious coloring problem, and the inclusion relations, for the classes of colinear graphs, linear graphs, and other subclasses of co-chordal and chordal graphs.

Note that since any $P_{6}$-free strongly chordal graph is a linear graph, it follows that split strongly chordal graphs form a subclass of linear graphs. Then, we can easily obtain that any split strongly chordal graph is a colinear graph, since if a graph $G$ is strongly chordal then $\bar{G}$ is also a strongly chordal graph.

\section{Harmonious Coloring on Colinear Graphs}

The formulation of the harmonious coloring problem in [5] is equivalent to the following formulation.

\section{Harmonious Coloring Problem}

Instance: Graph $G$, positive integer $K \leq|V(G)|$.

Question: Is there a positive integer $k \leq K$ and a proper coloring using $k$ colors such that each pair of colors appears together on at most one edge?

In this section we show that the harmonious coloring problem remains NPcomplete when restricted to the class of colinear graphs, which is a subclass of co-chordal graphs and a superclass of threshold graphs. The problem is NPcomplete on co-chordal graphs, since it is NP-complete on split graphs [1], and it has a polynomial solution on threshold graphs. Therefore, it is interesting to study the complexity of the problem on colinear graphs.

We first show that the problem remains NP-complete even when restricted to graphs which are simultaneously split graphs and undirected path graphs. Then, we show that every split undirected path graph is a colinear graph, thus, proving that the problem is NP-complete on colinear graphs. 
The following characterization of undirected path graphs will be used for obtaining our results. Note that, $\mathcal{C}$ denotes the set of all maximal cliques of a graph $G$; recall that, $C(v)$ denotes the set of all maximal cliques containing $v$.

Theorem 1. ([14,20]) A graph $G$ is an undirected path graph if and only if there exists a tree $T$ whose set of vertices is $\mathcal{C}$, so that for every vertex $v \in V(G)$, the subgraph $T[C(v)]$ of $T$ induced by the vertex set $C(v)$, is a path in $T$. Such a tree will be called characteristic tree of $G$.

We next show that the harmonious coloring problem is NP-complete for split undirected path graphs by exhibiting a reduction from the chromatic number problem for general graphs, which is known to be NP-complete [13].

Let $G$ be an arbitrary graph with $n$ vertices $v_{1}, v_{2}, \ldots, v_{n}$ and $m$ edges $e_{1}, e_{2}, \ldots, e_{m}$. We construct in polynomial time a split graph $\widehat{G}$, where $V(\widehat{G})=$ $K+I$, as follows: the independent set $I$ consists of $n$ vertices $\widehat{v}_{1}, \widehat{v}_{2}, \ldots, \widehat{v}_{n}$ which correspond to the vertices $v_{1}, v_{2}, \ldots, v_{n}$ of the graph $G$ and the clique $K$ consists of $m$ vertices $\widehat{u}_{1}, \widehat{u}_{2}, \ldots, \widehat{u}_{m}$ which correspond to the edges $e_{1}, e_{2}, \ldots, e_{m}$ of $G$. A vertex $\widehat{u}_{t} \in K, 1 \leq t \leq m$, is connected to two vertices $\widehat{v}_{i}, \widehat{v}_{j} \in I$, $1 \leq i, j \leq n$, if and only if the corresponding vertices $v_{i}$ and $v_{j}$ are adjacent in $G$. Note that, every $\widehat{u}_{i} \in K$ sees all the vertices of the clique $K$ and two vertices of the independent set $I$; thus, $|E(\widehat{G})|=\frac{m(m-1)}{2}+2 m$.

Moreover, we claim that the constructed split graph $\widehat{G}$ is also an undirected path graph. Indeed, we prove this by showing that the graph $\widehat{G}$ has a characteristic tree. Let $\mathcal{C}$ be the set of all maximal cliques of $\widehat{G}$. Note that $K$ is a maximal clique for $\widehat{G}$, thus, we have $|\mathcal{C}|=|I|+1$. Every vertex $\widehat{v}_{i} \in I$ belongs to exactly one maximal clique, i.e., $\left|C\left(\widehat{v}_{i}\right)\right|=1$. Additionally, every vertex $\widehat{u}_{i} \in K$ belongs to exactly three maximal cliques, one of which is maximal clique $K$, i.e., $\left|C\left(\widehat{u}_{i}\right)\right|=\left|N\left[\widehat{u}_{i}\right]\right|-|K|+1=3$.

Consider now a tree $T$ with vertex set $\mathcal{C}$, such that the maximal clique $K$ is connected by an edge to every maximal clique $C\left(\widehat{v}_{i}\right)$ for every $\widehat{v}_{i} \in I$, i.e., $T$ is a star. We now show that $T$ is a characteristic tree for $\widehat{G}$. Indeed, for every vertex $\widehat{v}_{i} \in I$, the subgraph $T\left[C\left(\widehat{v}_{i}\right)\right]$ induced by $C\left(\widehat{v}_{i}\right)$ is a path on one vertex, and also for every vertex $\widehat{u}_{i} \in K$, the subgraph $T\left[C\left(\widehat{u}_{i}\right)\right]$ is a path on three vertices. Therefore, the constructed graph $\widehat{G}$ has a characteristic tree and, thus, from Theorem 1 it follows that $\widehat{G}$ is a split undirected path graph.

We claim that the graph $G$ has a chromatic number $\chi(G)$ if and only if the split undirected path graph $\widehat{G}$ has a harmonious chromatic number $h(\widehat{G})=$ $\chi(G)+m$. Note that the same arguments are used in [1] for proving the NPcompleteness of the problem for split graphs.

Let $c_{i} \in\{1, \ldots, \chi(G)\}$ be the color assigned to the vertex $v_{i} \in G, 1 \leq i \leq n$, in a minimum coloring of $G$. We assign the color $c_{i}$ to the vertex $\widehat{v}_{i}$ of the set $I$ and a distinct color from the set $\{\chi(G)+1, \ldots, \chi(G)+m\}$ to each vertex of the clique $K$. Since two adjacent vertices of $G$ receive a different color, the neighbors of each $\widehat{u}_{i} \in K$ belonging to the independent set have distinct colors. Moreover, every vertex $\widehat{v}_{i} \in I$ sees $\left|N_{G}\left(v_{i}\right)\right|$ vertices of the clique $K$, where $N_{G}\left(v_{i}\right)$ is the neighborhood of the vertex $v_{i}$ in $G$. Thus, every pair of colors appears in at most 
one edge. In addition, the number of colors assigned to the set $I$ is equal to $\chi(G)$ and the number of colors assigned to the clique is equal to $m$. This results to a harmonious coloring of $\widehat{G}$ using $\chi(G)+m$ colors, which is minimum since the vertices of the set $I$ cannot receive a color assigned to a vertex of the clique $K$.

Conversely, a harmonious coloring of $\widehat{G}$ using $h(\widehat{G})=\chi(G)+m$ colors assigns $m$ colors to the vertices of the clique $K$ and $\chi(G)$ colors to the vertices of the set $I$. Note that, $\chi(G)$ is the minimum number of colors so that vertices $\widehat{v}_{i}, \widehat{v}_{j}$ having a neighbor in common are assigned different colors. Since $v_{i}, v_{j}$ are adjacent in $G$, it follows that we have a minimum coloring of $G$ using $\chi(G)$ colors.

Thus, we have proved the following result.

Theorem 2. The harmonious coloring problem is NP-complete for split undirected path graphs.

Next, we show the following result.

Theorem 3. Any split undirected path graph is a colinear graph.

Proof. Let $G$ be a split undirected path graph. Assume that $G$ is not a colinear graph. Then, from Definition 3 there exists an induced subgraph $G_{A}$ of $G$ such that $\lambda\left(\bar{G}_{A}\right) \neq \chi\left(G_{A}\right)$; thus, due to Proposition $1, \lambda\left(\bar{G}_{A}\right)>\chi\left(G_{A}\right)$.

From Theorem 1, we obtain that split undirected path graphs are hereditary, that is, every induced subgraph $G_{A}$ of $G$ is a split undirected path graph. Let $V\left(G_{A}\right)=K+I$ be a partition of the vertex set of $G_{A}$ into a maximal clique $K$ and an independent set $I$. Also, from Theorem 1 we have that $G_{A}$ has a characteristic tree $T$ with vertex set $\mathcal{C}$, where $\mathcal{C}$ is the set of all maximal cliques of $G_{A}$, such that for every vertex $v \in V\left(G_{A}\right)$, the subgraph $T[C(v)]$ of $T$ induced by the vertex set $C(v)$ is a path in $T$.

In particular, since $G_{A}$ is a split graph, for every vertex $v \in I$, the subgraph $T[C(v)]$ of $T$ induced by the vertex set $C(v)$ is a vertex in $T$ that corresponds to the unique maximal clique of $G_{A}$ that $v$ belongs to; we will denote this clique by $C_{v}$, i.e., $C_{v}=N_{G_{A}}[v]$ and $C(v)=\left\{C_{v}\right\}$ for every vertex $v \in I$. Also, for every vertex $v \in K$, the path $\left(C_{u}, \ldots, C_{x}, K, C_{y}, \ldots, C_{z}\right)$ of $T$ induced by the vertex set $C(v)$, always passes from the vertex $K$; equivalently, for every vertex $v \in K$, the subgraph of $T$ induced by the vertex set $C(v)$, corresponds to the vertex $K$ and to at most two vertex disjoint paths $\left(C_{y}, \ldots, C_{z}\right)$ and $\left(C_{x}, \ldots, C_{u}\right)$ where $C_{y}$ and $C_{x}$ are adjacent to $K$ in $T$. Moreover, observe that for any path $\left(K, C_{v_{1}}, C_{v_{2}}, \ldots, C_{v_{k}}\right)$ of the characteristic tree $T$ of $G_{A}$, we have $C_{v_{1}} \backslash\left\{v_{1}\right\} \supseteq$ $C_{v_{2}} \backslash\left\{v_{2}\right\} \supseteq \ldots \supseteq C_{v_{k}} \backslash\left\{v_{k}\right\}$, since $C_{v_{i}} \backslash\left\{v_{i}\right\}=N_{G_{A}}\left(v_{i}\right) \subset K$, where $v_{i} \in I$ for every $i, 1 \leq i \leq k$.

Let $\kappa: V\left(\bar{G}_{A}\right) \rightarrow\left\{1,2, \ldots, \lambda\left(\bar{G}_{A}\right)\right\}$ be a colinear coloring of $\bar{G}_{A}$. In order to see how a colinear coloring can be assigned to the vertices of $\bar{G}_{A}$ we refer to the colinear coloring algorithm presented in [17]. In particular, the algorithm first constructs the directed acyclic graph (DAG) $D_{\bar{G}_{A}}$ associated to the graph $\bar{G}_{A}$ and, then, finds a minimum path cover of the transitive DAG $D_{\bar{G}_{A}}$. The size of the minimum path cover of $D_{\bar{G}_{A}}$ equals the colinear chromatic number $\lambda\left(\bar{G}_{A}\right)$. Also, the algorithm assigns a colinear coloring $\kappa$ to the vertices of $\bar{G}_{A}$ 
such that a set of vertices are assigned the same color in $\kappa$ if and only if they belong to the same path of the minimum path cover of $D_{\bar{G}_{A}}$. Moreover, the DAG $D_{\bar{G}_{A}}$ associated to the graph $\bar{G}_{A}$ is constructed as follows: $V\left(D_{\bar{G}_{A}}\right)=V\left(\bar{G}_{A}\right)$ and $E\left(D_{\bar{G}_{A}}\right)=\left\{\overrightarrow{x y} \quad: \quad x, y \in V\left(D_{\bar{G}_{A}}\right)\right.$ and $\left.N_{\bar{G}_{A}}[x] \subseteq N_{\bar{G}_{A}}[y]\right\}$, where $\overrightarrow{x y}$ is a directed edge from $x$ to $y$. Note that $D_{\bar{G}_{A}}$ is a transitive DAG [17]. For simplicity, throughout the proof we will denote the DAG $D_{\bar{G}_{A}}$ associated to the graph $\bar{G}_{A}$ by $D$.

The following observations will be useful in the rest of this proof. Two vertices $u, v \in V(D)$ are not adjacent in $D$ if and only if neither $N_{\bar{G}_{A}}[v] \subseteq N_{\bar{G}_{A}}[u]$ nor $N_{\bar{G}_{A}}[v] \supseteq N_{\bar{G}_{A}}[u]$; we call two sets with this property incompatible. In $\bar{G}_{A}$ the vertices of $I$ form a clique, therefore, for two vertices $u, v \in I, u$ and $v$ are not adjacent in $D$ if and only if the sets $N_{\bar{G}_{A}}[u] \cap K$ and $N_{\bar{G}_{A}}[v] \cap K$ are incompatible. Note that, for any two vertices $u, v$ of $G_{A}, N_{\bar{G}_{A}}[u] \subseteq N_{\bar{G}_{A}}[v]$ if and only if $N_{G_{A}}(u) \supseteq N_{G_{A}}(v)$. Additionally, for every vertex $u \in I$, we have $N_{G_{A}}(u) \subset K$.

Having assumed that $\lambda\left(\bar{G}_{A}\right)>\chi\left(G_{A}\right)=|K|$, there exists a minimum path cover of $D$ with size $\lambda\left(\bar{G}_{A}\right) \geq|K|+1$. The size of a minimum path cover of $D$ equals the cardinality of a maximum independent set $I_{D}$ of $D$ [15]; thus, $\left|I_{D}\right| \geq|K|+1$. Moreover, the independent set $I_{D}$ corresponds to a collection $C$ of mutually incompatible sets $N_{\bar{G}_{A}}[v]$, for all $v \in I_{D}$, that is, $C=\left\{N_{\bar{G}_{A}}[v]: v \in\right.$ $\left.I_{D}\right\}$. Thus, $|C| \geq|K|+1$ and the sets of $C$ contain at most $|K|$ vertices of $K$. Also, recall that for any two vertices $u, v \in V(D)$ such that $u \in K$ and $v \in I$, if $u v \in E\left(\bar{G}_{A}\right)$ then $N_{\bar{G}_{A}}[u] \subset N_{\bar{G}_{A}}[v]$; thus, for any two vertices $u, v \in V(D)$ such that $u \in K$ and $v \in I, u$ and $v$ are adjacent in $\bar{G}_{A}$ if and only if $u$ and $v$ are adjacent in $D$.

Assume that $K \subset I_{D}$. Then, no vertex $v \in I$ can belong to $I_{D}$ since every vertex of $I$ is adjacent to at least one vertex of $K$ in $\bar{G}_{A}$ and, thus, in $D$, due to our assumption that $K$ is a maximal clique of $G_{A}$. Thus, not every vertex of $K$ can belong to $I_{D}$, since $\left|I_{D}\right| \geq|K|+1$. Assume that a vertex $u \in K$ belongs to $I_{D}$. Then, no vertex $v \in I$ that is adjacent to $u$ in $D$ and, thus, in $\bar{G}_{A}$, belongs to $I_{D}$; equivalently, $u \notin N_{\bar{G}_{A}}[v]$, for every vertex $v \in I_{D}$. Therefore, if we delete the vertex $u \in K$ from the set $I_{D}$, we obtain an independent set $I_{D}^{\prime}=I_{D} \backslash\{u\}$ and a collection $C^{\prime}=C \backslash\left\{N_{\bar{G}_{A}}[u]\right\}$ of at least $|K|$ mutually incompatible sets, which contain at most $|K|-1$ vertices of $K$. Using the same arguments, if we delete every vertex of $K$ from the independent set $I_{D}$, we obtain an independent set $I_{D}^{\prime \prime}$, such that $I_{D}^{\prime \prime} \subseteq I$ and $\left|I_{D}^{\prime \prime}\right| \geq k+1$ (where $k \leq|K|$ ), which corresponds to a collection $C^{\prime \prime}$ of at least $k+1$ mutually incompatible sets $N_{\bar{G}_{A}}[v], v \in I$, which contain at most $k$ vertices of $K$.

A collection $C^{\prime \prime}$ of at least $k+1$ mutually incompatible sets $N_{\bar{G}_{A}}[v], v \in I$, corresponds to a collection $F$ of at least $k+1$ mutually incompatible sets $N_{G_{A}}(v)$, $v \in I$. Since, for every vertex $v \in I$ we have $N_{G_{A}}(v)=C_{v} \backslash\{v\}$, it follows that a collection $F$ of at least $k+1$ mutually incompatible sets $N_{G_{A}}(v), v \in I$, corresponds to a collection of at least $k+1$ maximal cliques $C_{v}$ of $G_{A}, v \in I$, each of which must belong to a different path $\left(K, C_{v_{1}}, C_{v_{2}}, \ldots, C_{v_{k}}\right)$ of a characteristic tree $T$ of $G_{A}$. However, every vertex $z \in K$ belongs to at most two such paths, 


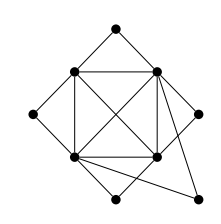

Fig. 3. A split graph $G$ which is not a colinear graph, since $\chi(G)=4$ and $\lambda(\bar{G})=5$. Also, $G$ is not an undirected path graph.

therefore, every vertex $z \in K$ belongs to at most two sets of the collection $F$. Thus, every vertex $z \in K$ belongs to at least $\left|C^{\prime \prime}\right|-2$ sets of the collection $C^{\prime \prime}$.

Summarizing, we have a collection $C^{\prime \prime}$ of at least $k+1$ mutually incompatible sets $N_{\bar{G}_{A}}[v], v \in I$, which contain at most $k$ vertices of $K$ and, also, every vertex $z \in K$ belongs to at least $\left|C^{\prime \prime}\right|-2$ sets of the collection $C^{\prime \prime}$. Recall that for two vertices $u, v \in I$, the sets $N_{\bar{G}_{A}}[u]$ and $N_{\bar{G}_{A}}[v]$ are incompatible if and only if the sets $N_{\bar{G}_{A}}[u] \cap K$ and $N_{\bar{G}_{A}}[v] \cap K$ are incompatible. Therefore, we have a collection of at least $k+1$ mutually incompatible vertex sets on $k$ vertices. It is easy to see that it is impossible to find a collection of at least $k+1$ mutually incompatible sets on $k$ vertices, if every vertex belongs to at least $k$ sets of the collection. This is a contradiction to our assumptions. Therefore, $G$ is a colinear graph.

Note that, not any split graph is a colinear graph (for example see Fig. 3). From Theorems 2 and 3, we obtain the following result.

Corollary 1. The harmonious coloring problem is NP-complete on the class of colinear graphs.

\section{Harmonious Coloring on Split Strongly Chordal Graphs}

In this section we show that the harmonious coloring problem admits a polynomial solution on the class of split strongly chordal graphs. Strongly chordal graphs form a known subclass of chordal graphs [4,11] and were first introduced by Farber [11]. A graph is strongly chordal iff it admits a strong elimination ordering; a vertex ordering $\sigma=\left(v_{1}, v_{2}, \ldots, v_{n}\right)$ is a strong elimination ordering of a graph $G$ iff $\sigma$ is a perfect elimination ordering and also has the property that for each $i, j, k$ and $\ell$, if $i<j, k<\ell, v_{k}, v_{\ell} \in N\left[v_{i}\right]$, and $v_{k} \in N\left[v_{j}\right]$, then $v_{\ell} \in N\left[v_{j}\right][6,11]$.

Let us now give the definitions of a $k$-sun and an incomplete $k$-sun. An incomplete $k$-sun $S_{k}(k \geq 3)$ is a chordal graph on $2 k$ vertices whose vertex set can be partitioned into two sets, $U=\left\{u_{1}, u_{2}, \ldots, u_{k}\right\}$ and $W=\left\{w_{1}, w_{2}, \ldots, w_{k}\right\}$, so that $W$ is an independent set, and $w_{i}$ is adjacent to $u_{j}$ if and only if $i=j$ or $i=j+1(\bmod k)$; the graph $S_{k}(k \geq 3)$ is a $k$-sun if $U$ is a complete graph. 
The following characterization of strongly chordal graphs was proved by Farber [11] and turns up to be useful in obtaining a polynomial solution for the harmonious coloring problem on split strongly chordal graphs.

Proposition 3. (Farber [11]) A chordal graph $G$ is strongly chordal if and only if it contains no induced $k$-sun.

Note also that a bipartite graph $G$ is chordal bipartite if and only if the split graph obtained from $G$ by making one of its two color classes complete is strongly chordal [19].

Next, we present a polynomial solution for the harmonious coloring problem on split strongly chordal graphs. Before describing our algorithm, we first construct a graph $H_{G}$ from a split graph $G$, which we call neighborhood intersection graph of $G$, and we use it in the proposed algorithm.

The neighborhood intersection graph $H_{G}$ of a split graph $G$. Let $G$ be a split graph, and let $V(G)=K+I$ be a partition of its vertex set, where $K$ induces a clique in $G$ and $I$ induces an independent set. We first compute the open neighborhood $N_{G}(v)$ of each vertex $v \in I$ and, then, we construct the following graph $H_{G}$, which depicts all intersection relations among the vertices' open neighborhoods: $V\left(H_{G}\right)=I$ and $E\left(H_{G}\right)=\left\{x y \quad: \quad x, y \in I\right.$ and $N_{G}(x) \cap$ $\left.N_{G}(y) \neq \emptyset\right\}$. It is easy to see that the resulting graph $H_{G}$ is unique up to isomorphism.

The following result is important for proving the correctness of our algorithm.

Lemma 1. The neighborhood intersection graph $H_{G}$ of a split strongly chordal graph $G$ is a chordal graph.

Proof. Let $G$ be a split strongly chordal graph and let $H_{G}$ be the neighborhood intersection graph of $G$. We will show that $H_{G}$ is a chordal graph, i.e., that $H_{G}$ is a $C_{k}$-free graph, for every $k \geq 4$. Since $G$ is a split graph, there exists a partition of its vertex set $V(G)=K+I$, where $K$ induces a clique and $I$ induces an independent set in $G$. By the construction of $H_{G}$, there is a one to one correspondence between the vertices of $V\left(H_{G}\right)$ and the vertices of $V(G) \cap I$.

Assume that $H_{G}$ is not a chordal graph and let $C_{k}=\left(v_{1}, v_{2}, \ldots, v_{k}\right)$ be a chordless cycle of $H_{G}$ on $k$ vertices, $k \geq 4$; thus, $v_{i} v_{j} \in E\left(H_{G}\right)$ if and only if $j=i+1(\bmod k)$. Therefore, we have that $N_{G}\left(v_{i}\right) \cap N_{G}\left(v_{j}\right) \neq \emptyset$ if and only if $j=i+1(\bmod k)$ or, equivalently, there exists at least one vertex $w_{i} \in K$ in $G$ such that $w_{i} \in N_{G}\left(v_{i}\right) \cap N_{G}\left(v_{j}\right)$ if and only if $j=i+1(\bmod k)$; note that, the set $W=\left\{w_{1}, w_{2}, \ldots, w_{k}\right\}$ consists of distinct vertices, since $C_{k}$ is a chordless cycle. Thus, $U=\left\{v_{1}, v_{2}, \ldots, v_{k}\right\}$ induces an independent set in $G$, $W=\left\{w_{1}, w_{2}, \ldots, w_{k}\right\}$ induces a clique in $G$, and $w_{i}$ is adjacent to $v_{j}$ if and only if $j=i$ or $j=i+1(\bmod k)$. Therefore, the subgraph of $G$ induced by the vertices $U \cup W$ is a $k$-sun, $k \geq 4$. It follows that $G$ is a split graph and, thus, it is a chordal graph, which contains a $k$-sun as an induced subgraph. This is a contradiction to our assumption that $G$ is a strongly chordal graph due to Proposition 3. Therefore, we conclude that $H_{G}$ is a chordal graph. 
The algorithm for a harmonious coloring of a split strongly chordal graph. The proposed algorithm computes a harmonious coloring and the harmonious chromatic number $h(G)$ of a split strongly chordal graph $G$, and works as follows:

Input: a split strongly chordal graph $G$, and a partition of its vertex set $V(G)=$ $K+I$, where $I$ induces an independent set in $G$ and $K$ induces a clique.

(i) construct the neighborhood intersection graph $H_{G}$ of $G$.

(ii) compute a minimum proper vertex coloring $\kappa: V\left(H_{G}\right) \rightarrow\left\{1,2, \ldots, \chi\left(H_{G}\right)\right\}$, and the chromatic number $\chi\left(H_{G}\right)$, of the chordal graph $H_{G}$ (see e.g. [15]).

(iii) compute a coloring $\kappa^{\prime}: V(G) \rightarrow\{1,2, \ldots, h(G)\}$ of $G$, by assigning $\kappa^{\prime}(v)=$ $\kappa(v)$ to each vertex $v \in I$, and a distinct color $\kappa^{\prime}(v)$ from the set $\left\{\chi\left(H_{G}\right)+\right.$ $\left.1, \chi\left(H_{G}\right)+2, \ldots, \chi\left(H_{G}\right)+|K|\right\}$ to each vertex $v \in K$.

(iv) return the value $\kappa^{\prime}(v)$ for each vertex $v \in V(G)$ and the size $\chi\left(H_{G}\right)+|K|$ of the number of different colors used in $\kappa^{\prime}$; the coloring $\kappa^{\prime}$ is a harmonious coloring of $G$, and $\chi\left(H_{G}\right)+|K|$ equals the harmonious chromatic number $h(G)$ of $G$.

Correctness of the algorithm. Let $G$ be a split strongly chordal graph, and let $V(G)=K+I$ be a partition of its vertex set, where $I$ induces an independent set in $G$ and $K$ induces a clique. Let $H_{G}$ be the neighborhood intersection graph of $G$.

We claim that the split strongly chordal graph $G$ has a harmonious chromatic number $h(G)=|K|+r$, where $r$ equals the chromatic number $\chi\left(H_{G}\right)$ of the graph $H_{G}$. Indeed, a harmonious coloring of $G$, using $h(G)=|K|+r$ colors, assigns a distinct color from the set $\{1,2, \ldots,|K|\}$ to each vertex of the clique $K$, and also assigns $r$ colors to the vertices of the set $I$. Note that, $r$ is the minimum number of colors so that vertices $v_{i}, v_{j} \in I$ having a neighbor in common are assigned different colors. Since $v_{i}, v_{j}$ are adjacent in $H_{G}$, it follows that $r$ is the minimum number of colors for which a proper vertex coloring of $H_{G}$ exists, i.e., $r=\chi\left(H_{G}\right)$.

Therefore, the split strongly chordal graph $G$ has a harmonious chromatic number $h(G)=|K|+\chi\left(H_{G}\right)$, where $\chi\left(H_{G}\right)$ is the chromatic number of the neighborhood intersection graph $H_{G}$ of $G$. Additionally, it is easy to see that the coloring $\kappa^{\prime}$ computed by the algorithm is a harmonious coloring of $G$ using $h(G)=|K|+\chi\left(H_{G}\right)$ colors.

Complexity of the algorithm. Let $G$ be a split strongly chordal graph on $n$ vertices and $m$ edges. Let $V(G)=K+I$ be a partition of its vertex set into a clique $K$ and an independent set $I$, and let $H_{G}$ be the neighborhood intersection graph of $G$. Step (i) of the algorithm, which includes the construction of the graph $H_{G}$, takes $O\left(n^{3}\right)$ time. Step (ii) computes a minimum proper vertex coloring of $H_{G}$; since from Lemma $1, H_{G}$ is a chordal graph, the problem is solvable in $O\left(n+m^{\prime}\right)$ time (see e.g. [15]), where $m^{\prime}=\left|E\left(H_{G}\right)\right|=O\left(n^{2}\right)$. Finally, both Steps (iii) and (iv) can be executed in $O(n)$ time. Therefore, the complexity of the algorithm is $O\left(n^{3}\right)$ time.

Therefore, the following result holds. 
Theorem 4. The harmonious coloring problem has a polynomial solution on split strongly chordal graphs.

\section{Concluding Remarks}

In this paper we show that the harmonious coloring problem is NP-complete on the classes of split undirected path graphs and colinear graphs. We also present a polynomial solution for the same problem on the class of split strongly chordal graphs. The interest of this result lies on the fact that the harmonious coloring problem is NP-complete on split graphs and strongly chordal graphs. In addition, polynomial solutions for the problem are only known for the classes of threshold graphs and connected quasi-threshold graphs; note that, the harmonious coloring problem is NP-complete on disconnected quasi-threshold graphs. Since linear graphs form a superclass of both split strongly chordal graphs and quasi-threshold graphs, the harmonious coloring problem is NP-complete on disconnected linear graphs, while it still remains open on connected linear graphs.

\section{References}

1. K. Asdre, K. Ioannidou, and S.D. Nikolopoulos. The harmonious coloring problem is NP-complete for interval and permutation graphs. Discrete Applied Math., 155:2377-2382, 2007.

2. K. Asdre and S.D. Nikolopoulos. NP-completeness results for some problems on subclasses of bipartite and chordal graphs. Theoret. Comput. Sci., 381:248-259, 2007.

3. H.L. Bodlaender. Achromatic number is NP-complete for cographs and interval graphs. Inform. Proc. Lett., 31:135-138, 1989.

4. A. Brandstädt, V.B. Le, and J.P. Spinrad. Graph Classes: A Survey. SIAM, Philadelphia, PA, 1999.

5. N. Cairnie and K. Edwards. Some results on the achromatic number. J. Graph Theory, 26:129-136, 1997.

6. G.J. Chang. Labeling algorithms for domination problems in sun-free chordal graphs. Discrete Applied Math., 22:21-34, 1988.

7. V. Chvátal and P.L. Hammer. Aggregation of inequalities for integer programming. Ann. Discrete Math., I:145-162, 1977.

8. Y. Civan and E. Yalçin. Linear colorings of simplicial complexes and collapsing. J. Comb. Theory A, 114:1315-1331, 2007.

9. K.J. Edwards. The harmonious chromatic number and the achromatic number. In R.A. Baily, editor, Surveys in Combinatorics, pages 13-47. Cambridge University Press, Cambridge, 1997.

10. K.J. Edwards and C. McDiarmid. The complexity of harmonious coloring for trees. Discrete Applied Math., 57:133-144, 1995.

11. M. Farber. Characterizations of strongly chordal graphs. Discrete Math., 43:173$189,1983$.

12. M. Farber. Domination, independent domination, and duality in strongly chordal graphs. Discrete Applied Math., 7:115-130, 1984. 
13. M.R. Garey and D.S. Johnson. Computers and Intractability: A Guide to the Theory of NP-completeness. W.H. Freeman, San Francisco, 1979.

14. F. Gavril. A recognition algorithm for the intersection graph of paths of a tree. Discrete Math., 23:377-388, 1978.

15. M.C. Golumbic. Algorithmic Graph Theory and Perfect Graphs. Academic Press, New York, 1980. Second edition, Annals of Discrete Mathematics 57, Elsevier, 2004.

16. K. Ioannidou and S.D. Nikolopoulos. Colinear coloring and colinear graphs. Technical Report TR-2007-06, Department of Computer Science, University of Ioannina, 2007.

17. K. Ioannidou and S.D. Nikolopoulos. Colinear coloring on graphs. In 3rd Annual Workshop on Algorithms and Computation (WALCOM'09), volume 5431, pages 117-128. LNCS, 2009.

18. D. Kratsch. Finding dominating cliques efficiently, in strongly chordal graphs and undirected path graphs. Discrete Math., 86:225-238, 1990.

19. T.A. McKee and F.R. McMorris. Topics in Intersection Graph Theory. Society for Industrial and Applied Mathematics, Philadelphia, 1999.

20. C.L. Monma and V.K. Wei. Intersection graphs of paths of a tree. J. Comb. Theory B, 41:141-181, 1986.

21. A.A. Schäffer. A faster algorithm to recognize undirected path graphs. Discrete Applied Math., 43:261-295, 1993.

22. M. Yannakakis and F. Gavril. Edge dominating sets in graphs. SIAM J. Applied Math., 38:364-372, 1980. 